\title{
Comparison of methods to determine auroral ionospheric conductances using ground-based optical and riometer data
}

\author{
A. Senior, M. J. Kosch, and F. Honary \\ Dept. of Communication Systems, Lancaster University, Lancaster, LA1 4WA, UK \\ Received: 20 June 2008 - Revised: 2 September 2008 - Accepted: 21 October 2008 - Published: 2 December 2008
}

\begin{abstract}
Ground-based images of auroral optical emissions and cosmic radio noise absorption provide information on particle precipitation which enhances ionospheric conductances. Knowledge of this conductance field is important to understand the current systems associated with auroral features. Three methods of using ground-based optical and riometer data to estimate ionospheric conductances in the aurora are compared to conductances derived from incoherent scatter radar measurements. It is shown that a method using the $557.7 \mathrm{~nm}$ emission intensity alone gives the best results for the Pedersen conductance whilst a method using both this intensity and cosmic noise absorption is best for the Hall conductance. A method using cosmic noise absorption alone gives reasonable performance for the Hall conductance and the Hall/Pedersen conductance ratio, but performs poorly for the Pedersen conductance. It also appears to underestimate the Hall conductance significantly during times when softer precipitation is present, for example in discrete auroral arcs. There is some indication that the methods do not degrade noticeably for angles up to $\sim 20^{\circ}$ off magnetic zenith.
\end{abstract}

Keywords. Ionosphere (Auroral ionosphere; Electric fields and currents; Ionosphere-magnetosphere interactions)

\section{Introduction}

In studies of the aurora, it is important to be able to understand the spatial structure of the electrical current systems which couple the magnetosphere and ionosphere. The currents can be measured in situ by spacecraft, or inferred from ground-based magnetometer measurements. However, the former cannot "image" the two-dimensional distribution of currents (without many spacecraft) and the latter is insensitive to the curl-free part of the current system (Amm, 1997).
The currents can be indirectly determined from Ohm's Law if the electric field and conductivity distributions are known. The former can be obtained from ground-based coherent scatter radars (Greenwald et al., 1978, 1995) on global and meso-scales $(\sim 10-100 \mathrm{~km})$. The latter can be obtained on global scales by inverting spacecraft measurements of auroral emissions (e.g. Aksnes et al., 2006), but these lack the resolution for meso-scale studies around individual auroral arcs. In those cases, ground-based measurements of optical emissions and cosmic radio noise absorption (CNA) give information on the precipitating electrons which create the ionisation leading to conductivity and these can be used to determine the meso-scale conductivity distribution.

In this study, three different methods of determining the ionospheric height-integrated conductivities (conductances) from optical and CNA measurements are compared. As a reference, the conductances derived from EISCAT incoherent scatter radar measurements are used. All three methods were originally calibrated by such measurements, but the data sets used here were not used in those calibrations and in that sense provide an independent test. The main aim of this study is to investigate under what circumstances each method performs well or poorly and consequently what might be required to form a better approach.

The study is restricted in that only measurements close to the magnetic field-line direction (magnetic zenith) are considered. Hence, ambiguities between horizontal and vertical structure in single-viewpoint images of the aurora are largely avoided. These could be a significant limitation of the methods in practice. Approaches based on tomographic inversion of multiple-viewpoint data are needed to overcome this (Nygrén et al., 1996; Gustavsson, 1998; Janhunen, 2001).

Correspondence to: A. Senior

(a.senior@lancaster.ac.uk)

Published by Copernicus Publications on behalf of the European Geosciences Union. 


\section{The methods tested}

The three methods under test in this study are summarised as follows.

\subsection{Statistical relationships using CNA alone (S07)}

Senior et al. (2007) presented a set of statistical relationships between cosmic noise absorption and the Hall and Pedersen conductances and their ratio for five different sectors of MLT. The relationships were derived by comparing conductances calculated from EISCAT measurements with CNA measurements from the nearby imaging riometer at Kilpisjärvi in Finland. Senior et al. (2007) fitted power-law functions to the data arguing that these seemed most suited to the data in the absence of any simple physical argument to support another form of function. The relationships had the most explaining power for the Hall conductance. Hereinafter, this method will be referred to as "S07".

2.2 Statistical relationship using $557.7 \mathrm{~nm}$ intensity alone (K98)

On the basis that the $557.7 \mathrm{~nm}$ column emission rate (intensity) is proportional to the flux of precipitating electrons and that the Pedersen conductance is proportional to the squareroot of this flux, Kosch et al. (1998b) fitted the conductance $\left(\Sigma_{P}\right.$ in $S$ ) to the square-root of the intensity ( $I$ in rayleighs):

$\Sigma_{P}=0.34+0.18 \sqrt{I}$

The study considered both pre- and post-magnetic midnight time sectors and found no significant difference in the relationship between these sectors. The data sets used spanned $\sim 19.5-05.5$ MLT with most of the data in the 21.5-03.5 MLT interval. Interestingly, Senior et al. (2007) found that the conductance-CNA relationships were more-or-less constant during the 19:00-04:00 MLT interval.

\section{3 "Energy map" method (K01)}

Kosch et al. (2001) combined both CNA and the $557.7 \mathrm{~nm}$ column intensity to estimate the characteristic energy of electron precipitation. Their approach was to use measurements to calibrate a simple physical model of the CNA and optical intensity in terms of the characteristic energy for assumed Maxwellian or exponential precipitating electron spectra. The objective was to produce high spatial-resolution maps of characteristic energy from imaging riometer and all-sky camera images, hence the description "energy map". As the method gives only the energy and not the flux, only the Hall/Pedersen conductance ratio can be directly determined by this approach. Here, the method is extended to determine the flux for the case of a Maxwellian spectrum.

Kosch et al. (2001) made the assumption that the $38.2 \mathrm{MHz} \mathrm{CNA}$ ( $A$ in $\mathrm{dB}$ ) is proportional to the square-root of the flux of precipitating electrons with energies exceeding
$25 \mathrm{keV}\left(\Phi_{25}\right): A=k \Phi_{25}$. The differential number flux $\phi$ (in $\mathrm{cm}^{-2} \mathrm{~s}^{-1} \mathrm{keV}^{-1}$ ) for a Maxwellian spectrum is

$\phi=\frac{\Phi}{E_{0}^{2}} E \exp \left(-E / E_{0}\right)$

where $E$ is the energy in $\mathrm{keV}, \Phi$ is the integral flux in $\mathrm{cm}^{-2} \mathrm{~s}^{-1}$ and $E_{0}$ is the characteristic energy in $\mathrm{keV}$. The differential flux peaks when $E=E_{0}$. The flux of electrons with energies exceeding $25 \mathrm{keV}$ is then

$$
\begin{aligned}
\Phi_{25} & =\int_{25}^{\infty} \frac{\Phi}{E_{0}^{2}} E \exp \left(-E / E_{0}\right) \mathrm{d} E \\
& =\Phi\left(1+\frac{25}{E_{0}}\right) \exp \left(-\frac{25}{E_{0}}\right)
\end{aligned}
$$

and hence

$A=k \sqrt{\Phi\left(1+\frac{25}{E_{0}}\right) \exp \left(-\frac{25}{E_{0}}\right)}$

where $k$ is a constant to be determined.

The dataset used by Kosch et al. (2001) gives values of $\Phi$ and $E_{0}$ from the inversion of the EISCAT electron density profiles and the corresponding CNA data measured by the IRIS riometer (see Sect. 3.1). The fluxes used by Kosch et al. (2001) are differential in pitch angle; to convert these to fluxes integral in pitch angle over the downward hemisphere as used here, the Kosch et al. (2001) fluxes are multiplied by $2 \pi$. Figure 1 presents the data and the leastsquares fit of Eq. (4). Note that, in keeping with Kosch et al. (2001), points where the CNA was less than $0.07 \mathrm{~dB}$ have been excluded. The relationship is reasonably linear with a Pearson correlation coefficient of 0.8 . The fit gives $k=2.4 \times 10^{-4} \mathrm{~dB} \mathrm{~cm} \mathrm{~s}^{1 / 2}$. This result appears to be reasonably consistent with the empirical relations quoted in Table 1 of Hargreaves (1969) when the difference in riometer frequency ( $30 \mathrm{MHz}$ versus $38.2 \mathrm{MHz}$ ) and minimum energy ( $40 \mathrm{keV}$ versus $25 \mathrm{keV}$ ) is accounted for.

Having established this relationship and given measurements of the CNA and $557.7 \mathrm{~nm}$ intensity the integral flux $\Phi$ can be determined by first finding $E_{0}$ using Eq. (18) of Kosch et al. (2001):

$\log _{e} \frac{A}{\sqrt{I}}=1.0-\frac{8.8}{E_{0}}$

where $I$ is the $557.7 \mathrm{~nm}$ intensity in $\mathrm{kR}$ and then solving Eq. (4) for $\Phi$. Finally, the Hall $\left(\Sigma_{H}\right)$ and Pedersen $\left(\Sigma_{P}\right)$ conductances can be estimated from $\Phi$ and $E_{0}$ by applying the formulae of Robinson et al. (1987):

$$
\begin{aligned}
\Sigma_{P} & =\frac{40 \bar{E}}{16+\bar{E}^{2}} \Phi_{E}^{1 / 2} \\
\frac{\Sigma_{H}}{\Sigma_{P}} & =0.45(\bar{E})^{0.85}
\end{aligned}
$$

These require the mean energy $(\mathrm{keV})$ and the energy flux (erg $\mathrm{cm}^{-2} \mathrm{~s}^{-1}$ or $\mathrm{mW} \mathrm{m}^{-2}$ ), which for the Maxwellian case, 


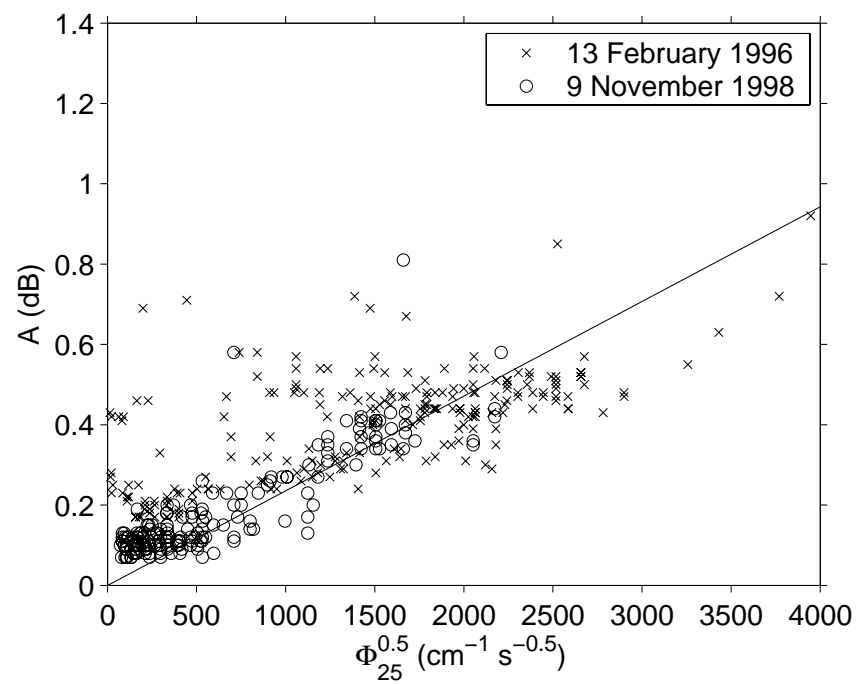

Fig. 1. Scatter plot of absorption $A$ versus the square-root of the flux of electrons with energies exceeding $25 \mathrm{keV}\left(\Phi_{25}\right)$ for the Kosch et al. (2001) data. The line is the least-squares fit of Eq. (4) to all points.

are given by $\bar{E}=2 E_{0}$ and $\Phi_{E} \approx 1.6 \times 10^{-9} \times 2 E_{0} \Phi$, respectively, taking account of the units of $\Phi$. Hereinafter, this method will be referred to as "K01". The original Kosch et al. (2001) method has been evaluated by Ashrafi et al. (2005).

\section{Observations}

\subsection{Instrumentation}

The locations of all the instruments used in this study are shown in Fig. 2. The EISCAT UHF incoherent scatter radar is located at Ramfjordmoen, near Troms $\varnothing$, Norway $\left(69.58^{\circ} \mathrm{N}, 19.22^{\circ} \mathrm{E}\right.$; Rishbeth and van Eyken, 1993). In this study we use two intervals of data, one from 8/9 March 1999 (Case 1) and the other from 23 November 2006 (Case 2). These intervals were selected due to the conjunction of available data from the radar and the all-sky imager and riometer used and also because they contain significant auroral activity over several hours. In Case 1, the radar was operating in the Common Programme 2 mode, where the antenna is scanned between four different look directions with a dwell time of $\sim 90 \mathrm{~s}$ on each position, making a 6-min cycle. The look directions are vertical, magnetic field-aligned (azimuth $183.2^{\circ}$, elevation $\left.77.2^{\circ}\right),\left(166.5^{\circ}, 62.9^{\circ}\right)$ and $\left(133.3^{\circ}, 60.4^{\circ}\right)$. The data have been integrated over the periods when the antenna was stationary in each direction. In Case 2, the radar was directed field-aligned $\left(185.1^{\circ}, 77.5^{\circ}\right)$ and the data were integrated in $60 \mathrm{~s}$ blocks.

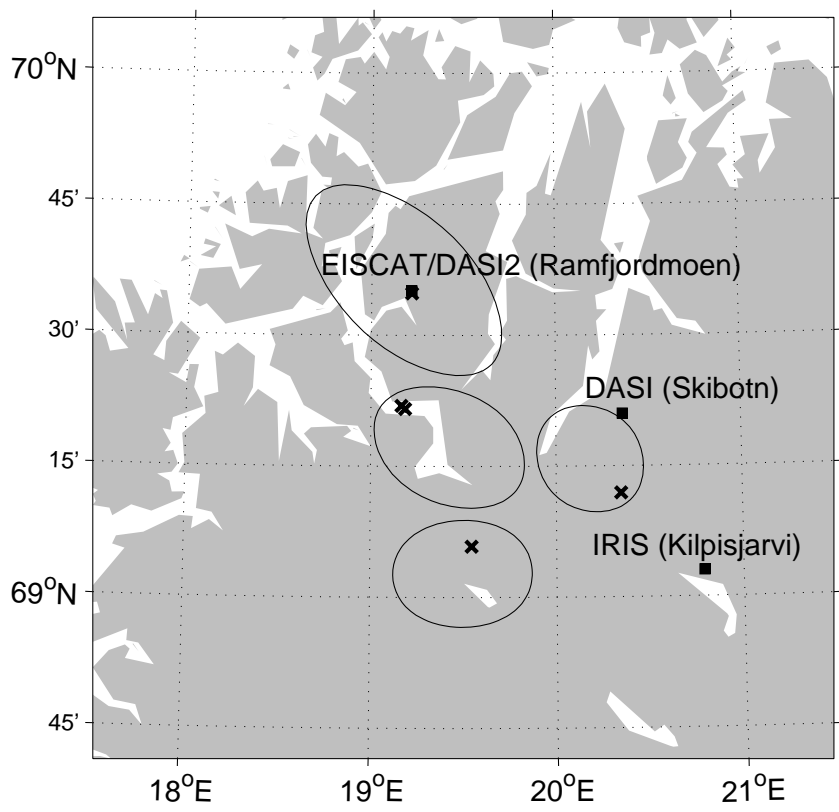

Fig. 2. The locations of the instruments used in this study, marked by the squares. The rings indicate the $-3 \mathrm{~dB}$ contours of four IRIS beams at $90 \mathrm{~km}$ altitude. The crosses mark the locations of the EISCAT radar beam at $110 \mathrm{~km}$ for all the scan positions used (note that the vertical position overlaps the instrument location).

The CNA measurements come from the IRIS imaging riometer at Kilpisjärvi, Finland $\left(69.05^{\circ} \mathrm{N}, 20.79^{\circ} \mathrm{E}\right.$; Browne et al., 1995). The riometer produces a $7 \times 7$ array of beams of widths (full width at $-3 \mathrm{~dB}$ ) between $11^{\circ}$ and $14^{\circ}$, corresponding to $18 \mathrm{~km}$ horizontally at $90 \mathrm{~km}$ altitude at the zenith. The field-of-view at $90 \mathrm{~km}$ is approximately $200 \times 200 \mathrm{~km}$. The locations of the beams relevant for this study are shown in Fig. 2. The fundamental temporal resolution is $1 \mathrm{~s}$.

In Case 1, $557.7 \mathrm{~nm}$ intensities are taken from the Digital All-Sky Imager (DASI) at Skibotn, Norway $\left(69.35^{\circ} \mathrm{N}\right.$, $20.36^{\circ}$ E; Kosch et al., 1998a). DASI records a $557.7 \mathrm{~nm}$ image every $10 \mathrm{~s}$ with a $10 \mathrm{~s}$ integration time and processed in realtime into a $10 \times 10 \mathrm{~km}$ grid at an assumed height of $100 \mathrm{~km}$. In Case 2, $557.7 \mathrm{~nm}$ intensities are taken from the Digital All-Sky Imager Mk. 2 (DASI2), located at the EISCAT site at Ramfjordmoen. DASI2 uses almost the same optics as DASI, but includes a filter-wheel and an efficient backilluminated CCD detector. In normal operations, a $256 \times 256$ pixel $557.7 \mathrm{~nm}$ image is taken every $15 \mathrm{~s}$ with an integration time of $1 \mathrm{~s}$. At $100 \mathrm{~km}$ altitude, the resolution is approximately $1 \times 1 \mathrm{~km}$ at the zenith which is similar to the crosssection of the EISCAT radar beam. 


\subsection{Data processing}

Since electron precipitation is geomagnetic field-aligned, it makes sense to compare measurements along the same magnetic field line as closely as possible. Due to the different instrument locations and look-directions, this was achieved approximately as follows.

The reference field-line was defined as the one on which the EISCAT radar beam intersected an altitude of $110 \mathrm{~km}$. This height was chosen as intermediate between the Pedersen and Hall conductance layers. The location of this intersection was then found in AACGM co-ordinates (formerly PACE; Baker and Wing, 1989). In this system, all points on the same field line have the same latitude and longitude. The $557.7 \mathrm{~nm}$ and CNA images were transformed into AACGM co-ordinates assuming a typical altitude for the $557.7 \mathrm{~nm}$ emission of $100 \mathrm{~km}$ (Kosch et al., 1998b) and a typical altitude for the absorbing layer of $90 \mathrm{~km}$ (Hargreaves, 1969). The images were then linearly interpolated to the coordinates of the field-line previously defined. In fact, since the geomagnetic field is inclined only about $12^{\circ}$ to the vertical, the horizontal alignment error in using geographic coordinates instead of AACGM would only be $4 \mathrm{~km}$ between the nominal conductance and CNA altitudes, smaller than the resolution of IRIS and DASI.

Matching of the data in time was achieved by integrating the camera and riometer measurements over each radar integration period. In the case of DASI2, where the image integration time is much less than the interval between images, this means that the integration does not take place over the complete radar integration period.

In Case 1, the $557.7 \mathrm{~nm}$ intensities were scaled up by a factor of 2.8. Without this scaling, the K98 method gave Pedersen conductance estimates that agreed closely in the shape of the time series, but were too small in magnitude compared to the radar-derived values. Additionally, the K01 method underestimated the characteristic energy and hence the Hall/Pedersen conductance ratio (the individual conductance estimates were also incorrect) compared to the radar. This single scaling factor, applied to the entire Case 1 data set, corrected this problem. The need for this scaling is not fully understood, but possibly there was a calibration problem with the DASI instrument at this time. A comparison of stellar intensities in an adjacent white-light imager between Case 1 and another night with clear sky showed no significant difference, suggesting that thin cloud was not to blame. This discrepancy does not seem to have been found by Ashrafi et al. (2005) who looked at data before and after the date in question which suggests it was a temporary problem. No such scaling was required in Case 2 which uses the newer DASI2 instrument for which the calibration had recently been determined using both stellar intensities and radioluminescent sources with good agreement.

The EISCAT plasma parameters were used to calculate the Hall and Pedersen conductivities and these were height- integrated over the altitude range $92-200 \mathrm{~km}$ to give the corresponding conductances in the manner described by Senior et al. (2007). The $557.7 \mathrm{~nm}$ intensities and CNA were used to estimate the conductances according to the three methods under test.

There are a number of possible sources of error in the data. Random error in the radar measurements contributes to random error in the conductances. The contribution to this from the error in the electron density is easy to assess. In Case 1, for the Hall conductance, $57 \%$ (96\%) of points have errors less than $1 \%(5 \%)$; for the Pedersen conductance the corresponding proportions are $84 \%$ and $100 \%$ and for the conductance ratio, $38 \%$ and $96 \%$. In Case 2, the proportions are $0 \%$ and $97 \%$ for the Hall conductance; $0 \%$ and $99 \%$ for the Pedersen conductance and $0 \%$ and $95 \%$ for the ratio. The errors are sufficiently small that one-standard-error error-bars on the plots are almost invisible, except for a few cases in the conductance ratio. Here, only the error bars for the ratio are presented. These error estimates are all underestimates because the contribution to the error from the uncertainty in the temperature measurements, which is more complicated to assess, is not included.

Besides random errors, there may also be systematic errors. Firstly, as the radar beam was not always directed fieldaligned and the $557.7 \mathrm{~nm}$ emission and CNA do not come from thin layers at fixed heights, there are ambiguities in the spatial correspondence of the measurements. Secondly, temporal ambiguities can arise because the quantities measured by the different instruments have different response time constants to changes in the precipitating particle flux. Thirdly, there can be systematic errors in the radar data analysis due to assumptions such as the ion composition being incorrect. The latter can also affect the calculation of the conductances. Furthermore, as the radar coverage stops at about $92 \mathrm{~km}$ altitude, the Hall conductance may be underestimated as the Hall conductance region can extend below this altitude.

\subsection{Case 1: 8/9 March 1999}

Figure 3 shows the observations from 8/9 March 1999 (18:30-03:00). All times herein are in UT unless otherwise stated. The geomagnetic $K_{p}$ indicies during the period were $2^{+}$(18:00 UT), $4^{+}$(21:00 UT), $4^{-}$(00:00 UT) and 4 (03:00 UT). Although the underlying $557.7 \mathrm{~nm}$ intensity and CNA data are not shown in the figure, the $557.7 \mathrm{~nm}$ intensity peaked at $10.3 \mathrm{kR}$ at $21: 16$ and had a minimum of $68 \mathrm{R}$ at 21:48. The CNA peak was $3.7 \mathrm{~dB}$ at 22:10 and the minimum was $-0.2 \mathrm{~dB}$ at 18:43. Gaps in all the time series (more evident in Case 2) are due to radar transmitter outages. Since the CNA and $557.7 \mathrm{~nm}$ intensity data were integrated over the actual radar data integrations, an absence of radar data leads to an absence of all data. Other blank spaces in the electron density plot are due to the radar analysis software failing to fit the plasma parameters to the measurement. Additional 

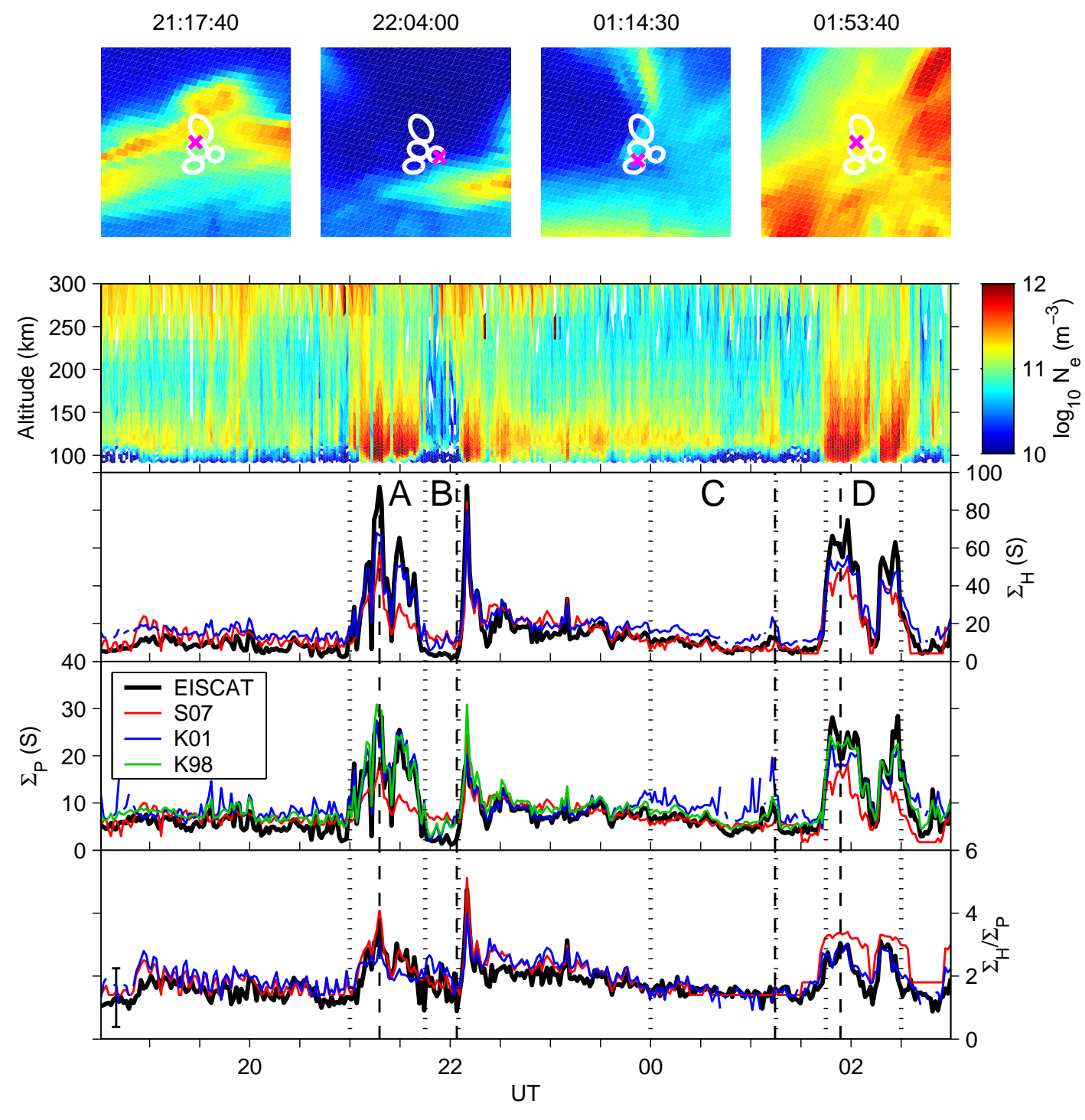

Fig. 3. Data from 8/9 March 1999 (Case 1). The methods are identified by the line colours which are labelled in the fourth row. The dotted vertical lines mark intervals of interest which are labelled in the third row. The dashed vertical lines mark the times of the images in the top row. Top row: $557.7 \mathrm{~nm}$ images. The intensity scale covers the range of the data in each image and the intensities have been compressed by taking the square-root to reveal weaker features. The white rings indicate the $-3 \mathrm{~dB}$ contours of a subset of IRIS beams. The pink crosses indicate the EISCAT UHF beam direction. The images are presented in AACGM co-ordinates (latitudes 64.83-67.83, longitudes 100.62-106.62) with north to the top and east to the right. Second row: electron densities from the EISCAT UHF radar. Third row: Hall conductances. Fourth row: Pedersen conductances. Bottom row: Hall/Pedersen conductance ratio. The one standard error bound is shown by error bars where the error exceeds 0.25 .

gaps in the time series for the K01 method are discussed in Sect. 4.3.

The image at 21:17:40 was taken close to the peak of the conductance enhancement in interval A. It shows a roughly east-west aligned arc. The structures in the arc appear to have scales similar to the size of the IRIS beams. The electron density profiles are enhanced over the height range 90$200 \mathrm{~km}$ (sometimes higher) during interval A. Both the S07 and K01 methods underestimate the Hall conductance dur- ing this interval, the former especially so. The S07 method also underestimates the Pedersen conductance significantly, though it reproduces the Hall/Pedersen ratio rather well.

The image at 22:04:00 occured near the end of interval B where all the methods under test appear to overestimate the conductances. The region of measurement is in an area of low $557.7 \mathrm{~nm}$ emission with an arc to the east. The electron densities are very low in interval B, also suggesting a lack of precipitation. In this interval, all three methods overestimate 

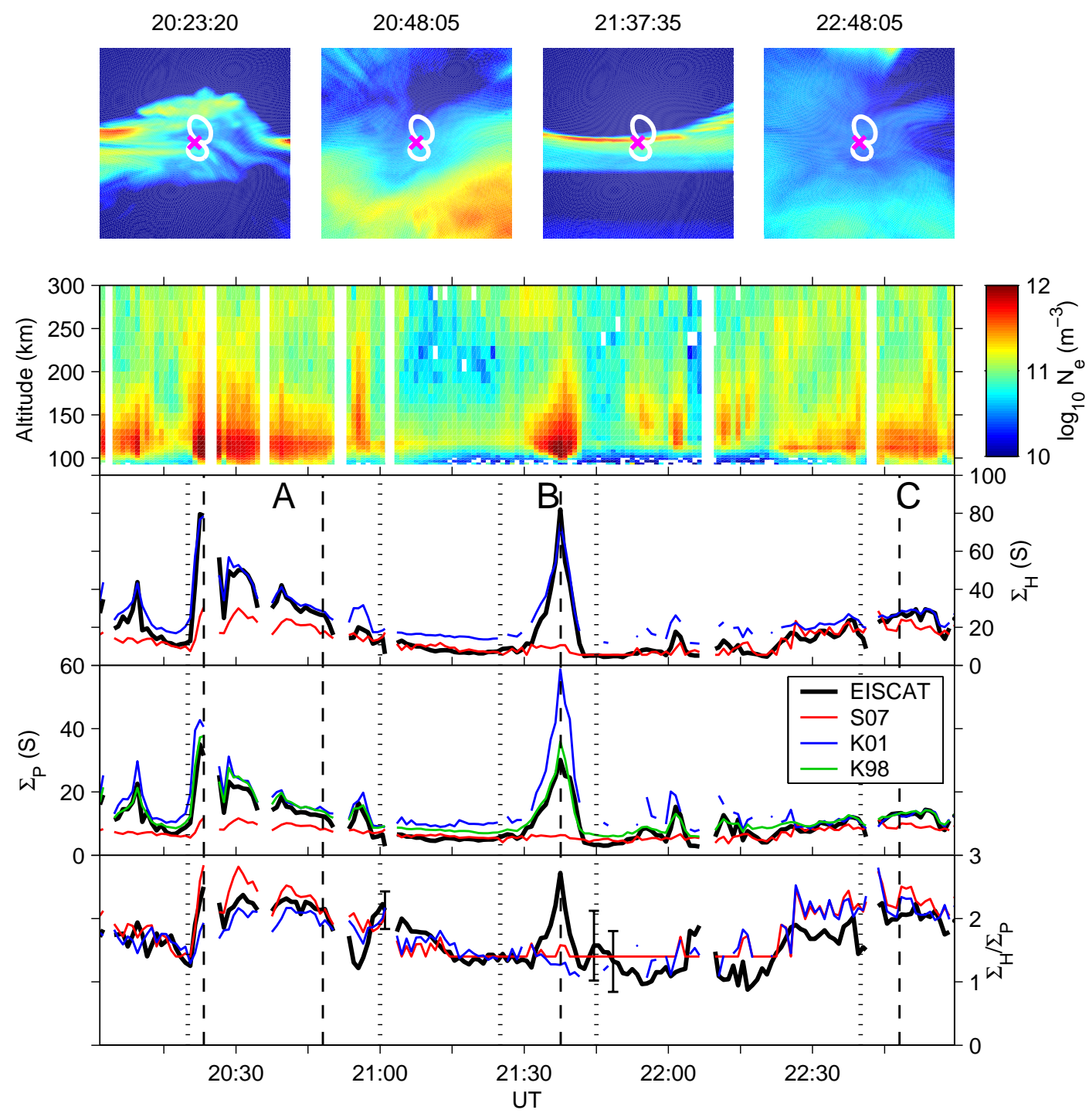

Fig. 4. Data from 23 November 2006 (Case 2). The format is as for Fig. 3.

the conductances. The S07 estimate of the Hall/Pedersen ratio is again rather good.

The image at 01:14:30 was taken near the end of interval $\mathrm{C}$ when the overestimate of the Pedersen conductance by the K01 method was greatest. The $557.7 \mathrm{~nm}$ emission shows some discrete structure of a similar scale-size to the IRIS beams. The electron density profiles are enhanced mainly in the $100-150 \mathrm{~km}$ interval with occasional enhancements to higher altitudes. Here, the S07 method estimates the conductances and their ratio quite well, but the K01 method overestimates the Hall and particularly the Pedersen conductances.

Finally the image at 01:53:40 was taken near the peak of the first conductance enhancement in interval D. Images covering the full camera field-of-view (not shown) show that the bright emission is associated with an "omega band" structure (Opgenoorth et al., 1983) of which successive waves cause the two enhancements in interval D. The image shows some structuring of the $557.7 \mathrm{~nm}$ emission in the bright region. The electron density profiles show considerable enhancement over altitudes from $90-200 \mathrm{~km}$ or higher. In interval D, all three methods underestimate the conductances, especially the S07 method, which also overestimates the Hall/Pedersen conductance ratio.

\subsection{Case 2: 23 November 2006}

Figure 4 shows the observations from 23 November 2006 (20:00-23:00). The $K_{p}$ indicies during this period were $3^{+}$ (18:00 UT) and 4 (21:00 UT). The peak $557.7 \mathrm{~nm}$ intensity was $43.0 \mathrm{kR}$ at 20:23 and the minimum was $947 \mathrm{R}$ at 22:06. The peak CNA was $0.8 \mathrm{~dB}$ at 20:23 and the minimum was $0 \mathrm{~dB}$ at 22:13. 
In interval $\mathrm{A}$, there is a series of diminishing conductance enhancements. The K01 method estimates these well, whereas the S07 method greatly underestimates the first one, progressively getting better during the interval. The image at 20:23:20 is close to the peak of the first enhancement. It shows a mixture of discrete arc structures and more diffuse emission. The image at 20:48:05 is close to the end of the interval where the conductance is diminishing. It shows that the IRIS beams view a region of diffuse emission with a steady gradient across the beams.

The image at 21:37:35 is close to the peak of the conductance enhancement in interval B. It shows that an east-west arc lies close to magnetic zenith, covering one beam of IRIS but only grazing the other. The arc is particularly intense in a narrow $(\sim 6 \mathrm{~km})$ strip on its poleward edge. This strip is much narrower than the IRIS beam dimensions. In this interval, the S07 method completely fails to estimate the large enhancement in conductivity associated with this arc. The K01 method performs well for the Hall conductance but overestimates the Pedersen conductance by about a factor of two. Neither the S07 nor the K01 methods correctly estimate the enhancement in the conductance ratio.

The image at 22:48:05 occurs near the start of interval C. Similarly to the end of interval A, the IRIS beams view an area of diffuse emission. The electron density profile at this time is similar in shape to that at the end of interval A. Here, the K01 method performs well for the conductances and is reasonable for their ratio. The S07 method slightly underestimates the conductances and overestimates their ratio.

As in Case 1, the K98 method performs very well throughout the whole interval. Recall that in Case 2 no scaling was applied to the $557.7 \mathrm{~nm}$ intensities.

\section{Discussion}

Firstly, explanations for the discrepancies between the results of methods under test and the EISCAT-derived conductances are considered, followed by a statistical summary of the performance of the methods.

\subsection{Shape of the precipitating electron spectrum}

A common feature in both cases 1 (intervals $\mathrm{A}$ and $\mathrm{D}$ ) and 2 (interval A) is a tendency for the S07 method to underestimate the conductances and to overestimate their ratio during periods when soft precipitation is present, as indicated by enhanced electron densities at higher altitudes (above $150 \mathrm{~km}$ ). Enhanced Pedersen and Hall conductances and CNA correspond to enhanced precipitating electron fluxes in the approximate energy bands $2-10,5-50$ and $>20 \mathrm{keV}$, respectively. There is thus considerable overlap between the bands enhancing the Hall conductance and CNA, but if there is preferential enhancement to the soft end of the spectrum, the Hall conductance may be enhanced without further enhance-

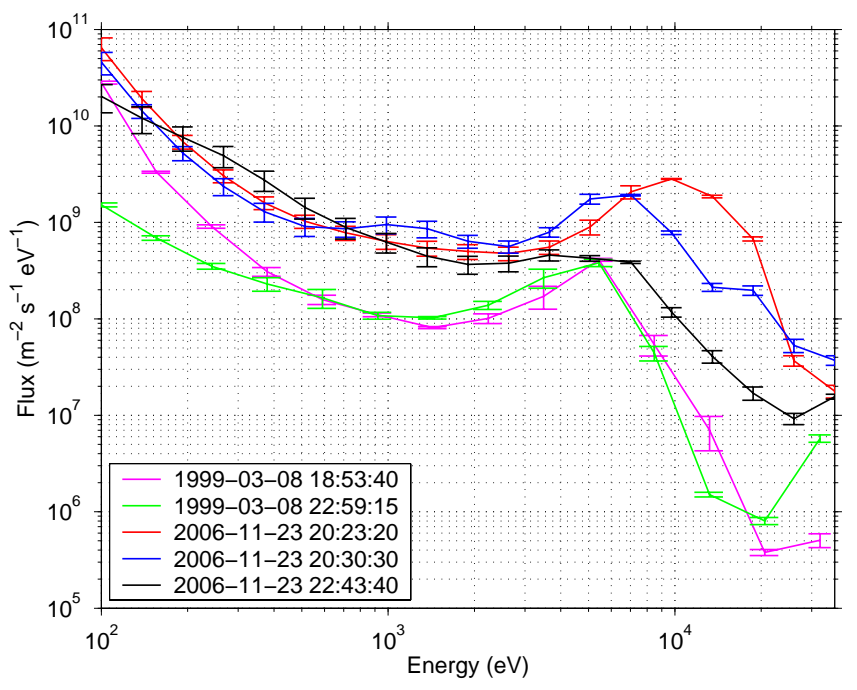

Fig. 5. Precipitating electron energy spectra derived from the EISCAT electron density profiles at the times shown. Error bars on each curve indicate the one standard error bound.

ment of CNA. The situation for the Pedersen conductance is even clearer as there is no significant overlap in energy band with CNA. Therefore, enhanced soft precipitation leads to an underestimate of conductances by the S07 method because the CNA contains no information on the soft part of the spectrum. The Hall/Pedersen conductance ratio is overestimated for a similar reason.

Figure 5 shows precipitating electron spectra derived from the EISCAT electron density profiles using the method of Semeter and Kamalabadi (2005). The error estimates were produced by running the inversion 40 times for each input density profile, randomly varying the densities within the error estimates provided by the radar analysis and taking the standard deviation of the resulting fluxes in each energy bin. Systematic errors could result from the neutral atmosphere model used (MSISE-90) or the effective recombination coefficient, which was taken as the empirical form of Vickrey et al. (1982). The CNA was nearly the same at the three times shown for Case 2 (23 November 2006), giving the same estimate of the conductances by the S07 method, but the conductances derived from the electron density profiles are quite different. The discrepancy between the two estimates becomes less at each successive time until they agree closely for the final case at 22:43:40. In the first case (20:23:20) the densityderived conductances greatly exceed the S07 estimate. The spectrum for this time shows a strong peak in flux at $10 \mathrm{keV}$ which should lead to enhancement of both the Hall and Pedersen conductances, but is too soft to greatly enhance the absorption. Indeed, at the higher energies $(>20 \mathrm{keV})$ the flux is much closer to that in the other two cases. The final case (22:43:40) shows only a small local flux maximum around $4 \mathrm{keV}$ so that the conductances are relatively less enhanced 
compared to the CNA. The middle case (20:30:30) is intermediate both in the discrepancy between the estimates and in the shape of the spectrum. There is a clear local flux maximum around $7 \mathrm{keV}$ but the high-energy tail looks rather similar to that of the final case but with a higher flux. Note that as the UHF radar measurements stop at $\sim 92 \mathrm{~km}$ altitude, the full extent of the height interval and hence energy range relevant to CNA is not covered by the data.

As the S07 method is a statistical model, one would expect to find times where overestimation occurs too. Examples of this are around 18:53 and 22:59 in Case 1. At these times, both the S07 and K01 methods overestimate the Hall conductance and Hall/Pedersen ratio but are somewhat closer for the Pedersen conductance. Electron spectra for these times are shown in Fig. 5 and are very similar. Overall, the fluxes are lower than in the examples from Case 2, but the notable feature is the steeper fall off in flux above the local peak at $\sim 5 \mathrm{keV}$. This would tend to result in less enhancement of the Hall conductance than would be the case for the spectrum shapes of the Case 2 examples.

\subsection{Limited spatial resolution}

In interval B in Case 2 (Fig. 4), an arc with an intense narrow edge passed through the radar beam revealing a strong enhancement of the conductances. The $557.7 \mathrm{~nm}$ emission was strongly enhanced and the K98 method reproduced the Pedersen conductance rather well. However, the S07 method failed to reproduce the enhancement and the K01 method greatly overestimated the Pedersen conductance. The common factor between $\mathrm{S} 07$ and $\mathrm{K} 01$ is the CNA. It is clear from the $557.7 \mathrm{~nm}$ images that the arc has a thickness which is small compared to the IRIS beams and the poleward beam is almost completely in a region of no emission. If the CNA has a similar morphology, it is not surprising that IRIS shows little response in this case, even though the CNA could be locally strong in the arc. The spatial scale of the CNA measurement will almost integrate away the localised enhancement. This underestimate of CNA is consistent with the underestimate by the S07 method.

The observed $557.7 \mathrm{~nm}$ intensity at $21: 37: 35$ was $39 \mathrm{kR}$ and the CNA was $0.09 \mathrm{~dB}$. The radar-derived Hall conductance was $82 \mathrm{~S}$ and the Pedersen conductance $30 \mathrm{~S}$. The corresponding $\mathrm{K} 01$ estimates are 75 and $59 \mathrm{~S}$, respectively. It can be shown that if the CNA had been $2 \mathrm{~dB}$, the K01 method would estimate the conductances as $\Sigma_{H}=81 \mathrm{~S}$ and $\Sigma_{P}=30 \mathrm{~S}$, so that the Hall conductance estimate is only slightly reduced whereas the Pedersen conductance is greatly reduced and matches the radar-derived value. Hence an underestimate of CNA due to having too-large a measurement scale could account for the failure of the K01 method in this case.

\subsection{Other issues}

In interval B of Case 1, all three methods overestimate the Hall and Pedersen conductances, though the S07 method estimates their ratio quite well. Pi2 pulsations observed by the SAMNET magnetometer array (Yeoman et al., 1990) indicate a substorm onset just prior to $21: 45$, the start of interval B. In the DASI images (not shown except for 22:04 in Fig. 3), the $557.7 \mathrm{~nm}$ emission is strongest to the equatorward edge of the field-of-view. Over interval B, the more intense $557.7 \mathrm{~nm}$ emission progresses poleward, but does not reach the EISCAT field-of-view until after interval B. DASI has a rather limited dynamic range ( 8 bits) and the intensifier gain is adjusted in realtime to compensate for this. A consequence is that when bright emission is present, the gain is reduced, effectively raising the noise level. It seems likely that the overestimate by $\mathrm{K} 98$ towards the end of interval B is a result of this effect. Regarding the IRIS CNA measurements, the CNA at the end of the interval was very low $(\sim 0.1 \mathrm{~dB})$ which is comparable to the error in the quiet-day cosmic noise power estimate (Senior et al., 2007).

In interval $\mathrm{C}$ of Case 1, the K01 method overestimates the Hall and Pedersen conductances yet estimates their ratio well. The origin of this behaviour can be traced to low values of CNA which fluctuate around zero; although it is unphysical, CNA can be negative as a result of an inaccurate estimate of the quiet-day noise power. An analysis of the K01 method shows that as the CNA approaches zero, so does the estimate of the characteristic energy. At the same time, the estimate of the integral number flux tends to infinity. If the CNA is zero or negative, the results become undefined. It is the delicate balance of this behaviour as the CNA tends to zero which leads to the large fluctuations, particularly in the Pedersen conductance estimate in interval C. However, since the conductance ratio depends only on the characteristic energy estimate which is well-behaved even for zero CNA, the ratio estimate is also well-behaved. The gaps in the line for the K01 method correspond to those points where the CNA is zero or negative.

\subsection{Statistical performance of the methods}

Table 1 gives the Pearson correlation coefficients between the conductance parameter estimates from the EISCAT measurements and from the three methods under test. It is clear that the K98 method performs best for the Pedersen conductance (although the K01 method is only slightly worse) and that the K01 method performs best for the Hall conductance. For the Hall/Pedersen conductance ratio, the S07 method seems to have an advantage over the K01 method, although in Case 1 this is only slight. The general good performance of the K01 method is probably a result of the combined use of the $557.7 \mathrm{~nm}$ intensity and CNA, which allows both the energy and flux of the precipitation to be estimated. 
In Case 1, the different radar look directions allow the variation in the performance of the methods with look direction to be explored. A comparison of the correlation coefficients for the results of the three methods with the EISCATderived conductances for each of the four radar look directions showed that at the $95 \%$ confidence level, there was no significant difference between radar look directions. For IRIS, the four directions correspond to approximate angles of $25^{\circ}-52^{\circ}$ off magnetic zenith; for DASI the range is $2^{\circ}-34^{\circ}$ and for the radar itself, the range is $0^{\circ}-23^{\circ}$. It is not possible to conclude that the performance of the methods under test is relatively unaffected by angle off zenith, at least up to the limits observed, because field-aligned reference measurements of the conductances are not available at each location, but at least the methods become no worse than the case of a non field-aligned radar measurement. Comparing the correlation coefficients with the results of Case 2 (Table 1) also suggested that the results are no worse (in fact sometimes better) than for the case where both the radar and imager have field-aligned look directions.

\subsection{Improvements to the methods}

The S07 method performs relatively poorly compared to the K01 and K98 methods, but this is hardly surprising when one quantity (CNA) is used to describe a system with more than one degree of freedom (the precipitating electron spectrum). Adding a second measurement as in the K01 method brings a considerable improvement. Surprisingly, the K98 method, also based on a single quantity, estimates another single quantity, the Pedersen conductance, remarkably well. The joint performance of the K01 and K98 methods suggests that there is little room for improvement to the basic method when instrumental effects such as spatial resolution and estimation of the riometer quiet-day level are taken away.

Temporal resolution was not investigated in this study, but modern optical imagers and riometers have good temporal resolution, comparable to or finer than the recombination time of the plasma at the altitudes of the conductance layers. Therefore the data should allow short timescale changes in conductance to be monitored, although the methods might require some adaptation to account for non-equilibrium effects.

\section{Conclusions}

The cases studied here allow the following conclusions to be drawn about the three methods tested:

1. The S07 method underestimates conductances when soft precipitation is present, for example inside auroral arcs. Overestimation is seen in some circumstances in diffuse aurora, depending on the detail of the precipitating electron spectrum.
Table 1. Pearson correlation coefficients between the conductance parameter estimates from EISCAT and from the three methods under test. The upper number in each case is for Case 1, the lower number is for Case 2.

\begin{tabular}{cccc}
\hline Parameter & K98 & K01 & S07 \\
\hline$\Sigma_{H}$ & & 0.98 & 0.87 \\
& & 0.97 & 0.63 \\
$\Sigma_{P}$ & 0.96 & 0.90 & 0.71 \\
& 0.97 & 0.88 & 0.55 \\
$\Sigma_{H} / \Sigma_{P}$ & & 0.70 & 0.78 \\
& & 0.56 & 0.73 \\
\hline
\end{tabular}

2. Despite the above, the S07 method provided the best estimates of the Hall/Pedersen conductance ratio.

3. The K01 method provided the best estimates of the Hall conductance.

4. The K98 method provided the best estimates of the Pedersen conductance.

5. Insufficient spatial (and presumably temporal) resolution in the measurements can lead to serious errors in the conductance estimates.

6. There is no evidence for a deterioration in the performance of the methods at least up to magnetic zenith angles of $\sim 20^{\circ}$. This is a conservative estimate based on the maximum magnetic zenith angle of the radar measurement.

It is worth noting that the results show that when the estimation of "bulk" parameters such as the conductances are required, as opposed to height-resolved conductivities or the spectrum of precipitating particles, good results can be obtained using only one or two ground-based measurements.

Acknowledgements. This work was supported by grant PP/C000218/1 from the UK Particle Physics and Astronomy Research Council (now the Science and Technology Facilities Council (STFC)). IRIS and SAMNET are funded by STFC. EISCAT is an international association supported by research organisations in China (CRIRP), Finland (SA), France (CNRS, till end 2006), Germany (DFG), Japan (NIPR and STEL), Norway (NFR), Sweden (VR), and the United Kingdom (STFC). We are very grateful to B. Gustavsson (U. Troms $\varnothing$ ) and U. Brändström (IRF, Kiruna) for their assistance in calibrating the DASI2 instrument. We thank the reviewers for their constructive criticism of the manuscript.

Topical Editor M. Pinnock thanks R. Makarevich and A. Brekke for their help in evaluating this paper.

\section{References}

Aksnes, A., Stadsnes, J., Østgaard, N., Germany, G. A., Oksavik, K., Vondrak, R. R., Brekke, A., and Løvhaug, U. P.: Height 
profiles of the ionospheric electron density derived using spacebased remote sensing of UV and X ray emissions and EISCAT radar data: A ground truth experiment, J. Geophys. Res., 111, A02301, doi:10.1029/2005JA011331, 2006.

Amm, O.: Ionospheric elementary current systems in spherical coordinates and their application, J. Geomag. Geoelectr., 49, 947955, 1997.

Ashrafi, M., Kosch, M. J., and Honary, F.: Comparison of the characteristic energy of precipitating electrons derived from groundbased and DMSP satellite data, Ann. Geophys., 23, 135-145, 2005 ,

http://www.ann-geophys.net/23/135/2005/.

Baker, K. B. and Wing, S.: A new magnetic coordinate system for conjugate studies at high-latitudes, J. Geophys. Res., 94, 91399143, 1989.

Browne, S., Hargreaves, J. K., and Honary, B.: An Imaging Riometer for Ionospheric Studies, Electron. Commun. Eng., 7, 209$217,1995$.

Greenwald, R. A., Weiss, W., Nielsen, E., and Thomson, N. R.: STARE: A new radar auroral backscatter experiment in northern Scandinavia, Radio Sci., 13, 1021-1039, 1978.

Greenwald, R. A., Baker, K. B., Dudeney, J. R., Pinnock, M., Jones, T. B., Thomas, E. C., Villain, J. P., Cerisier, J. C., Senior, C., Hanuise, C., Hunsucker, R. D., Sofko, G., Koehler, J., Nielsen, E., Pellinen, R., Walker, A. D. M., Sato, N., and Yamagishi, H.: DARN/SUPERDARN: A global view of the dynamics of highlatitude convection, Space Sci. Rev., 71, 761-796, 1995.

Gustavsson, B.: Tomographic inversion for ALIS noise and resolution, J. Geophys. Res., 103, 26 621-26 632, 1998.

Hargreaves, J. K.: Auroral absorption of HF radio waves in the ionosphere: a review of results from the first decade of riometry, Proc. IEEE, 57, 1348-1373, 1969.

Janhunen, P.: Reconstruction of electron precipitation characteristics from a set of multi-wavelength digital all-sky auroral images, J. Geophys. Res., 106, 18 505-18 516, 2001.

Kosch, M. J., Hagfors, T., and Nielsen, E.: A new digital all-sky imager experiment for optical auroral studies in conjunction with the Scandinavian twin auroral radar experiment, Rev. Sci. Inst., 69, 578-584, 1998a.
Kosch, M. J., Hagfors, T., and Schlegel, K.: Extrapolating EISCAT Pedersen conductances to other parts of the sky using groundbased TV auroral images, Ann. Geophys., 16, 583-588, 1998b, http://www.ann-geophys.net/16/583/1998/.

Kosch, M. J., Honary, F., del Pozo, C. F., Marple, S. R., and Hagfors, T.: High-resolution maps of the characteristic energy of precipitating auroral particles, J. Geophys. Res., 106, $28925-$ $28937,2001$.

Nygrén, T., Markkanen, M., Lehtinen, M., and Kaila, K.: Application of stochastic inversion in auroral tomography, Ann. Geophys., 14, 1124-1133, 1996, http://www.ann-geophys.net/14/1124/1996/.

Opgenoorth, H. J., Oksman, J., Kaila, K. U., Nielsen, E., and Baumjohann, W.: Characteristics of eastward drifting omegabands in the morning sector of the auroral oval, J. Geophys. Res., 88, 9171-9185, 1983.

Rishbeth, H. and van Eyken, A. P.: EISCAT - Early history and the first ten years of operation, J. Atmos. Terr. Phys., 55, 525-542, 1993.

Robinson, R. M., Vondrak, R. R., Miller, K., Dabbs, T., and Hardy, D.: On calculating ionospheric conductances from the flux and energy of precipitating electrons, J. Geophys. Res., 92, 25652569, 1987.

Semeter, J. and Kamalabadi, F.: Determination of primary electron spectra from incoherent scatter radar measurements of the auroral $E$ region, Radio Sci., 40, RS2006, doi:10.1029/2004RS003042, 2005.

Senior, A., Kavanagh, A. J., Kosch, M. J., and Honary, F.: Statistical relationships between cosmic radio noise absorption and ionospheric electrical conductances in the auroral zone, J. Geophys. Res., 112, A11301, doi:10.1029/2007JA012519, 2007.

Vickrey, J. F., Vondrack, R. R., and Matthews, S. J.: Energy deposition by precipitating particles and Joule dissipation in the auroral ionosphere, J. Geophys. Res., 87, 5184-5196, 1982.

Yeoman, T. K., Milling, D. K., and Orr, D.: Pi2 pulsation polarisation patterns on the UK Sub-auroral Magnetometer Network (SAMNET), Planet. Space Sci., 38, 589-602, 1990. 